\title{
Postmarketing Surveillance of Suicidal Adverse Events with Pediatric Use of Antidepressants
}

\author{
Andrew D. Mosholder, M.D., M.P.H., and Carol A. Pamer, R.Ph.
}

\begin{abstract}
Objective: The aim of this analysis was to delineate trends in spontaneous postmarketing reporting data with antidepressant drugs for adverse events involving suicidal behaviors in children and adolescents.

Methods: The U.S. Food and Drug Administration (FDA) Adverse Event Reporting System (AERS) was searched for postmarketing adverse event reports of suicidal thoughts and behaviors occurring in children and adolescents treated with 10 antidepressant drugs. The search covered the period from market launch of each drug through November 2003.

Results: A total of 524 reports were returned by the search. All drugs had reports, and most drugs demonstrated 15 or fewer reports annually, with the following two exceptions. We observed a peak of reporting for fluoxetine in the early 1990s, and another peak of reporting for paroxetine in recent years. Further investigation revealed that the peak in recent paroxetine reporting was accounted for by reports from consumers, whereas reporting by health professionals remained fairly constant. In contrast, the earlier peak in reports for fluoxetine was not accounted for by an increase in consumer reporting.

Conclusions: Spontaneous reporting data for suicidal events in pediatric patients treated with antidepressant drugs appears to be highly variable and subject to various influences. The most appropriate method to assess an association of antidepressant drug treatment with suicidal behaviors is examination of systematically collected data with appropriate comparison groups, such as randomized, controlled trial data.
\end{abstract}

\section{INTRODUCTION}

I

$\mathrm{N}$ VIEW OF RECENT CONCERNS regarding a possible association between antidepressant drug treatment and suicidal ideation and behavior in pediatric patients (U.K. Committee on Safety of Medicines 2003), we examined spontaneous postmarketing reporting data with antidepressant drugs for adverse events involving suicidal behaviors in children and adolescents. The aim of this analysis was to delineate reporting trends by quantifying and graphically compar- ing these data for 10 different antidepressants. These data have been presented at an U.S. Food and Drug Administration (FDA) Advisory Committee meeting (Mosholder 2004) but have not been previously reported in the literature.

\section{METHODS}

The FDA Adverse Event Reporting System (AERS) is a searchable electronic database that stores data on adverse drug experiences re- 
ported with marketed pharmaceutical products. Sources of AERS case reports include health care professionals, pharmaceutical manufacturers, and health consumers. Our AERS database search included the following 10 antidepressant drugs marketed since the mid-1980s: Bupropion, citalopram, escitalopram, fluoxetine, fluvoxamine, mirtazapine, nefazodone, paroxetine, sertraline, and venlafaxine. We compiled a list of adverse event terms from the Medical Dictionary for Regulatory Activities (MedDRA*) that we felt would represent the spectrum of suicidal and self-injurious behaviors. Reports were selected from the AERS database if they contained at least one of the following 11 MedDRA terms: Depression suicidal, gunshot wound, intentional self-injury, nonaccidental overdose, overdose not otherwise specified, self-injurious behavior, self-injurious ideation, self-mutilation, suicidal ideation, suicide attempt, and completed suicide. All U.S. reports received by the FDA over the lifetime of the product, through November 2003, were included in the search. The age of the patient was limited to 17 years or under, and one of the se-

*Northrup Grumman Corporation, website www.meddramsso.com lected antidepressant drugs had to be listed as the primary suspect drug for the event. If a report had been forwarded to the FDA from the manufacturer, the date assigned to the report was the date it was received by the manufacturer. The source of the report (consumer or health professional) was determined from information provided within the report.

\section{RESULTS}

For the 10 drug products in their entire marketing period to date, there were 524 reports with at least one of the selected MedDra terms in patients with a reported age of 17 years and under. In order of decreasing numbers of reports, the following total numbers of reports were obtained: fluoxetine (210 reports), paroxetine (119), sertraline (76), bupropion (47), venlafaxine (34), citalopram (21), fluvoxamine (10), mirtazapine (5), nefazodone (1), and escitalopram (1). (Note that escitalopram was the most recently marketed among these drugs.) Figure 1 provides a graphical display of the AERS report totals by drug and year for the entire marketing period of each drug through November 2003.

The numbers of pediatric suicide-related reports received yearly for the drugs of interest

\begin{tabular}{|llll|}
\hline- Bupropion & $\rightarrow$ Citalopram & Escitalopram & $\rightarrow$ Fluoxetine \\
$\rightarrow$ Fluvoxamine & $\rightarrow$ Mirtazapine & + Nefazodone & - Paroxetine \\
- Sertraline & $\rightarrow$ Venlafaxine & & \\
\hline
\end{tabular}

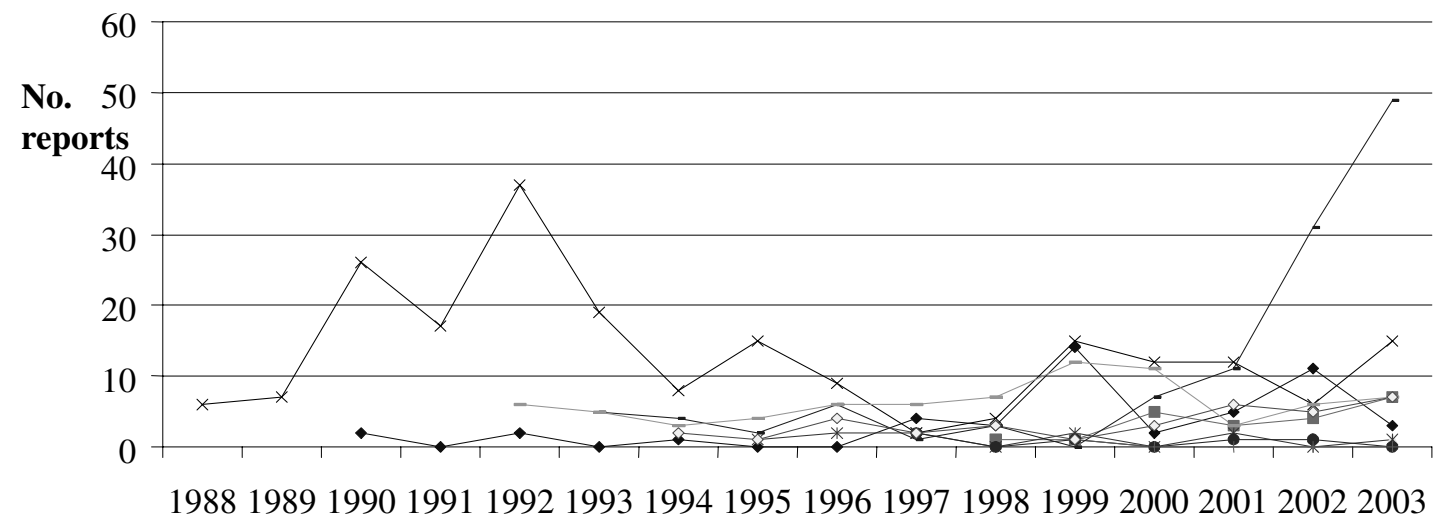

Year Received by Manufacturer/FDA

FIG. 1. Total raw counts of reports for pediatric suicide-related terms, by drug and year (uncorrected for duplicate reports). FDA = U.S. Food and Drug Administration. 


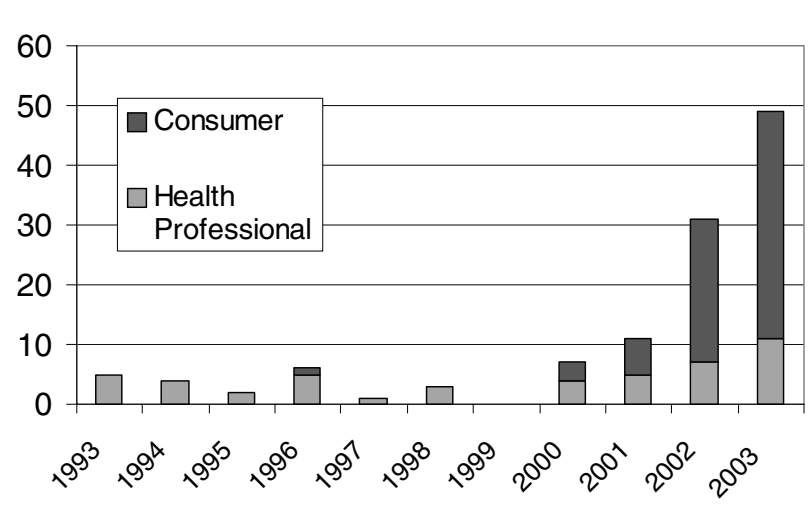

FIG. 2. Paroxetine: Raw counts of the FDA Adverse Event Reporting System (AERS) reports of pediatric suicidal events, by type of reporter and year.

in this class, as seen in Figure 1, have been approximately $10-15$ per year or less, with two prominent exceptions. Paroxetine exhibited a substantial increase in the numbers of reports beginning in calendar year 2002. Most cases in this recent increase in reports for paroxetine were submitted by non-health professionals (Fig. 2). Fluoxetine also exhibited a large peak in reports in the early 1990s, particularly in calendar year 1992. In contrast to the more recent paroxetine reports, the majority of these reports were submitted by health care professionals (data not shown).

Individual review of each of the cases was not performed owing to the volume of cases returned by the search, and so some cases may have been reported more than once. However, we did select a subsample of these case reports for hands-on review by choosing reports that were received during the first 3 years of marketing for the antidepressant involved. Among the 94 case reports selected by this criterion, nine reports were excluded from the review owing to the presence of database coding errors or reported events that could not be assessed as possible suicidal behavior. Seven of the remaining 78 cases were reported twice.

\section{DISCUSSION}

One of the limitations of this analysis was that we were able to conduct a hands-on review on only a subsample of the reports. However, it did not appear from our targeted hands-on review that there were an inordinate number of duplicated case reports.

With respect to the peak of reporting for fluoxetine in the early 1990s, it may be noted that in 1990, Tiecher et al. (Tiecher et al. 1990) published a case series describing 6 patients who developed intense suicidal ideation during treatment with the fluoxetine. Similar events were subsequently reported in pediatric patients by King et al. (King et al. 1991). The topic of antidepressant-induced suicidality was the subject of a Psychiatric Drugs Advisory Committee meeting in September 1991. Speculatively, reporting may have been increased by the publicity about whether fluoxetine treatment can induce suicidal behavior.

In the most recent 2 years of reporting that we analyzed, consumers reported the majority of the cases for paroxetine, which is a distinction from the previous pattern of reporting for that drug (Fig. 2). For comparison, in the AERS database as a whole, data from the first portion of 2003 showed that health care professionals submitted $66 \%$ of all reports. It seems likely that various influences came into play to account for this pattern of reporting. In 1998, Internet submission of MedWatch reports through the FDA website was implemented, which may have influenced the extent of consumer reporting. In October 2002, BBC News aired a Panorama program on adverse reactions to paroxetine, and in 2003 , it was reported that pediatric trial data indicated that paroxetine was associated with self-injurious behavior. Such events may have influenced the level of reporting. But, as with any ecological correlation, we cannot be certain that these apparent associations between publicity and increases in spontaneous reporting reflect a cause-and-effect relationship.

Spontaneous reporting data on adverse drug reactions have well-known limitations, including a large, but unknown, number of events that go unreported, and frequently incomplete clinical information contained in the reports (Ahmad 2003). In general, spontaneous postmarketing report data are most useful for distinctive or rare adverse drug reactions. Pemoline-induced liver injury is an example of the type of adverse drug reaction for which postmarketing surveillance data are critical. If cases of hepatotoxicity associated with pemoline treatment had not 
been reported to the manufacturer or the FDA, the risk of severe hepatotoxicity with pemoline would not have been uncovered (Safer et al. 2001). Evaluating postmarketing surveillance data for psychiatric drugs associated with suicidal adverse events is problematic, however, because the outcome of interest (suicidal ideation or behavior) is also an outcome of the indication for the drugs (depression).

The elapsed time from marketing a new drug has been observed to influence the number of postmarketing adverse event reports. Typically, the highest level of reporting occurs in the earlier years of marketing; this phenomenon is known as the Weber effect (Weber 1984). It has also been reported that, for selective serotonin reuptake inhibitors (SSRIs), increased spontaneous reporting sometimes occurs following approval of a new indication (Hartnell et al. 2003). In our data, the numbers of suiciderelated adverse event reports for antidepressants received by the FDA appear to be influenced by publicity surrounding specific drugs.

\section{CONCLUSIONS}

In sum, the spontaneous reporting data for suicidal events in pediatric patients treated with antidepressant drugs show reports for all drugs surveyed. The level of reporting of these events appears to be highly variable and subject to various influences. The most appropriate method to assess an association of antidepressant drug treatment with suicidal behaviors is examination of systematically collected data with appropriate comparison groups, such as randomized, controlled trial data.

\section{DISCLOSURES}

Dr. Andrew D. Mosholder and Carol A. Pamer are employees of the U.S. Food and Drug Administration and have no financial relationships with pharmaceutical companies.

\section{ACKNOWLEDGMENT}

Grateful acknowledgement is made to Lynette Swartz, MBA/CIS, M.Ed., of the FDA's
Office of Drug Safety, for her assistance with statistical computing.

\section{REFERENCES}

Ahmad SR: Adverse drug event monitoring at the Food and Drug Administration: Your report can make a difference. J Gen Intern Med 18:57-60; 2003.

Committee on Safety of Medicines: Selective serotonin reuptake inhibitors (SSRIs): Overview of regulatory status and CSM advice relating to major depressive disorder (MDD) in children and adolescents, including a summary of available safety and efficacy data. December 10, 2003. Online document at: medicines.mhra.gov.uk/ ourwork/monitorsafequalmed/safetymessages / ssrioverview\%5F101203.htm

Hartnell NR, Wilson JP, Patel NC, Crismon ML: Adverse event reporting with selective serotonin reuptake inhibitors. Ann Pharmacother 37:13871391, 2003.

King RA, Riddle MA, Chappell PB, Hardin MT, Anderson GM, Lombroso P, Scahill L: Emergence of self-destructive phenomena in children and adolescents during fluoxetine treatment. J Am Acad Child Adolesc Psychiatry 30:179-186, 1991.

Mosholder A: Office of Drug Safety data resources for the study of suicidal events associated with pediatric use of antidepressants. February 2, 2004. Online document at: www.fda.gov/ ohrms/dockets/ac/04/slides/4006S1_06_Mosholder_files/frame.htm

Safer DJ, Zito JM, Gardner JF: Pemoline hepatotoxicity and postmarketing surveillance. J Am Acad Child Adolesc Psychiatry 40:622-629, 2001.

Tiecher MH, Glod C, Cole JO: Emergence of intense suicidal preoccupation during fluoxetine treatment. Am J Psychiatry 147:207-210, 1990.

Weber JCP: Epidemiology of adverse reactions to nonsteroidal anti-inflammatory drugs. In: Advances in Inflammation Research, volume 6. Edited by Rainsford KD, Velo GP. New York, Raven Press, 1984, pp 1-7.

Address reprint requests to: Andrew D. Mosholder, M.D., M.P.H. FDA Division of Drug Risk Evaluation Office of Drug Safety 10903 New Hampshire Ave. Mail Stop 3411 Silver Spring, MD 20993-0002

E-mail: andrew.mosholder@fda.hhs.gov 
Reproduced with permission of the copyright owner. Further reproduction prohibited without permission. 